\title{
Synovial Fluid of Patient With Rheumatoid Arthritis Enhanced Osmotic Sensitivity Through the Cytotoxic Edema Module in Synoviocytes
}

\author{
Min Jeong $\mathrm{Ji}^{1,2}$, Hee Jung Ryu ${ }^{3 *}$ and Jeong Hee Hong ${ }^{1 *}$ \\ ${ }^{1}$ Department of Physiology, College of Medicine, Lee Gil Ya Cancer and Diabetes Institute, Gachon University, Incheon, \\ South Korea, ${ }^{2}$ Department of Oral Biology, Yonsei University College of Dentistry, Seoul, South Korea, ${ }^{3}$ Division of \\ Rheumatology, Department of Internal Medicine, Gachon University College of Medicine Gil Medical Center, Incheon, \\ South Korea
}

OPEN ACCESS

Edited by:

Huijie Bian,

Fourth Military Medical University,

China

Reviewed by:

Curtis Okamoto,

University of Southern California,

United States

Kui Zhang,

Fourth Military Medical University,

China

*Correspondence:

Hee Jung Ryu

ryu2093@naver.com

Jeong Hee Hong

minicleo@gachon.ac.kr

Specialty section:

This article was submitted to

Membrane Traffic,

a section of the journal

Frontiers in Cell and Developmental

Biology

Received: 27 April 2021

Accepted: 28 July 2021

Published: 31 August 2021

Citation:

Ji MJ, Ryu HJ and Hong JH (2021) Synovial Fluid of Patient With

Rheumatoid Arthritis Enhanced

Osmotic Sensitivity Through

the Cytotoxic Edema Module

in Synoviocytes.

Front. Cell Dev. Biol. 9:700879. doi: 10.3389/fcell.2021.700879
Rheumatoid arthritis $(R A)$ is an autoimmune disease that causes inflammation of the synovial membrane ultimately leading to permanent damage in the affected joints. For this study, synovial fluids from 16 patients diagnosed with either RA or osteoarthritis $(\mathrm{OA})$ were used to examine volume regulation and cooperative water channels, both of which are involved in the cytotoxic edema identified in RA-fibroblast-like synoviocytes (FLS). The osmolarity and inflammatory cytokine interleukin (IL)-6 of synovial fluids from RA patients were mildly enhanced compared to that from OA patients. RAFLS demonstrated the enhanced property of regulatory volume increase in response to IL-6 and synovial fluids from RA patients. Although there was no difference in the protein expression of the volume-associated protein sodium-potassium-chloride cotransporter1 (NKCC1), its activity was increased by treatment with IL-6. Membrane localization of NKCC1 was also increased by IL-6 treatment. Additionally, both the protein and membrane expressions of aquaporin-1 were increased in RA-FLS by IL-6 stimulation. The IL-6-mediated enhanced osmotic sensitivity of RA-FLS likely involves NKCC1 and aquaporin-1, which mainly constitute the volume-associated ion transporter and water channel elements. These results suggest that RA-FLS provide enhanced electrolytes and concomitant water movement through NKCC1 and aquaporin-1, thereby inducing cellular swelling ultimately resulting in cytotoxic edema. Attenuation of cytotoxic edema and verification of its related mechanism will provide novel therapeutic approaches to RA treatment within the scope of cytotoxic edema.

Keywords: cytotoxic edema, interleukin-6, synovial fluid, NKCC1, aquaporin-1

\section{INTRODUCTION}

The pathology of rheumatoid arthritis (RA) as an autoimmune disease involves joint inflammation, pannus formation, and eventual joint destruction (Nanki et al., 2001). Fibroblast-like synoviocytes (FLS) enter an aggressive state that changes them to have tumor-like characteristics like anchorageindependent growth, resistance to apoptosis, and elevated rates of cellular proliferation in RA 
due to various proinflammatory mediators (Georganas et al., 2000; Deon et al., 2001). Of those proinflammatory mediators, interleukin (IL)-6 specifically is believed to mediate the pathogenesis of inflammatory arthritis including RA (Kokkonen et al., 2010; Hampel et al., 2013). Several studies suggest that the IL-6 level of synovial fluids revealed differences between the patients with RA and osteoarthritis (OA) (Kokebie et al., 2011; Machado Diaz et al., 2012; Hampel et al., 2013; Elicabe et al., 2017). Although its statistical difference was variable in their studies, the concentration of IL- 6 of RA was higher than that of OA. In hypertensive encephalopathy accompanying juvenile RA, it has been reported that enhanced levels of IL-6 are associated with cytotoxic edema (Takano et al., 2002). In addition, IL-6 expression is associated with the development of brain edema in patients with meningiomas (Park et al., 2010).

Change in cellular volume in response to hyper-/hypotonic stimulation is a physiological cellular response. Hypotonic stimulation triggers cell swelling through water influx into the cytosolic area and subsequent elicited regulatory volume decrease (RVD) as a homeostatic reaction. On the contrary, hypertonic stimulation mediates cell shrinkage, which causes regulatory volume increase (RVI) (Huang et al., 2019). This cellular ionic and volume homeostasis is maintained by various ion transporters, of which NKCC1 in particular has been implicated in osmotic cell swelling (Pedersen et al., 2006). NKCC1 is associated with cytotoxic edema in neuronal ischemic stroke and epilepsy (Xu et al., 2017). Although the enhanced IL-6 level in synovial fluids has been suggested and IL-6 is considered to be an important biomarker in RA (Kokebie et al., 2011), the pathogenesis of RA with a focus on cytotoxic edema remains unclear, and the role of NKCC1 and volume homeostasis in FLS remains to be defined.

Synovial fluid is known to contain several immune cells including mast cells, monocytes, synovial lining cells, and inflammatory cytokines, which together represent the degree of inflammation in the affected area (Oliviero et al., 2017). It has been previously shown that synovial fluid is a useful substrate in the diagnosis of joint diseases (Altobelli et al., 2017; Oliviero et al., 2017). In the present study using synovial fluid samples from patients with either RA or OA, we demonstrated IL-6mediated volume regulation and identified the cooperative water channels involved in cytotoxic edema in RA-FLS. Although RA typically presents with multiple symptoms, attenuation of cytotoxic edema and verification of its related mechanisms will provide new advanced therapeutic approaches for the treatment of RA-related edema.

\section{MATERIALS AND METHODS}

\section{Patient Enrollment and Assessment of Clinical Parameters}

A total 16 patients ( 8 patients with RA and 8 patients with knee OA) were enrolled, all of whom had effusion on knee joints. The patients with RA fulfilled the 2010 American College of Rheumatology (ACR)/European League Against Rheumatism (EULAR) classification criteria (Aletaha et al., 2010), and the patients with biological disease-modifying anti-rheumatic drugs (DMARDs) were excluded. The patients with primary knee OA as controls were previously diagnosed based on clinical and radiological examination. In the knee OA group, patients with secondary knee OA due to inflammatory arthritis or metabolic diseases and those with total knee replacements were excluded. All patients underwent medical history and joint examination. In patients with RA, the tender and swollen joint counts were examined, and erythrocyte sedimentation rate (ESR, $\mathrm{mm} / \mathrm{h})$ and C-reactive protein $(\mathrm{CRP}, \mathrm{mg} / \mathrm{dl})$ were measured at enrollment. The status of rheumatoid factor (RF, IU/ml) and anti-citrullinated peptide antibodies (anti-CCP, U/ml) were collected. Disease activities were checked by Disease Activity Score 28-ESR (DAS28ESR). In patients with knee OA, the severity of knee OA was evaluated by Kellgren-Lawrence (KL) from plain radiographs.

\section{Synovial Fluid Collection}

The synovial fluid $(\geq 10 \mathrm{ml})$ was obtained by therapeutic arthrocentesis with aseptic procedure. Synovial fluids were aspirated from affected knee joints after acupuncture using a 30$\mathrm{ml}$ syringe with 21-gauge needle. The synovial fluid was then centrifuged at 1,500 rpm for $15 \mathrm{~min}$.

\section{Reagents}

Recombinant human IL-6 (200-06) was purchased from Peprotech (Cranbury, NJ, United States). NKCC1 (ab59791) antibody was purchased from Abcam (Cambridge, United Kingdom). The phosphorylated form of OSR-1 (pOSR1, Ser325) antibody (07-2273) was purchased from Merck (Darmstadt, Germany). Peroxidase-conjugated $\beta$-actin antibody (A3854) was purchased from Sigma-Aldrich (St Louis, MO, United States). OSR-1 (\#3729) and IL-6 (\#12153) antibodies were purchased from Cell Signaling (Danvers, MA, United States). Aquaporin (AQP)-1 antibody (CSB-PA16085A0Rb) was purchased from CUSABIO (Houston, TX, United States). DAPI fluoromount- $\mathrm{G}^{\mathrm{TM}}$ mounting solution was purchased from Electron Microscopy Sciences (Electron Microscopy Sciences, Hatfield, PA, United States). 2'-7'-Bis-(carboxyethyl)-5-(and6)-carboxyfluorescein (BCECF-AM; \#0061) was purchased from Teflabs (Austin, TX, United States). Cell volume indicator calcein-AM (C1430) was purchased from Molecular Probes (Eugene, OR, United States). Amiloride (EIPA, Sigma-Aldrich; 1154-25-2) and other chemicals not mentioned here were purchased from Sigma-Aldrich.

\section{Primary Synoviocytes Culture and HEK293T Cell Culture}

Human FLS, isolated from normal synovial tissues (HFLS, 408-05a), RA-FLS (408RA-05a), and HFLS-osteoarthritis (OA) (408OA-05a) cells were purchased from Cell Application, Inc. (San Diego, CA, United States) (Jones et al., 2017; Wang et al., 2017). Cells were maintained in Dulbecco's modified Eagle's medium (Invitrogen, Carlsbad, CA, United States; 11995-065) containing 10\% fetal bovine serum (FBS, Invitrogen; 16000-044), and $100 \mathrm{U} / \mathrm{ml}$ penicillin-streptomycin (Invitrogen; 15140-122) and incubated at $37^{\circ} \mathrm{C}$ in $5 \% \mathrm{CO}_{2} / 95 \%$ air. When cells reached 
$80 \%$ confluency, the cell culture medium was aspirated, and the cells were washed with Dulbecco's phosphate-buffered saline (DPBS, Welgene, Daegu, South Korea; LB001-02), followed by treatment with trypsin/ethylene diamine tetraacetic acid (EDTA) for $1 \mathrm{~min}$. Cells were used within five passages for all experiments. Human HEK293T (ATCC ${ }^{\circledR}$, United States; CRL-3216 ${ }^{\text {TM }}$ ) cell was maintained by Dulbecco's modified Eagle's medium containing $10 \%$ fetal bovine serum (FBS) and $100 \mathrm{U} / \mathrm{ml}$ penicillinstreptomycin and incubated at $37^{\circ} \mathrm{C}$ in $5 \% \mathrm{CO}_{2} / 95 \%$ air.

\section{Enzyme-Linked Immunosorbent Assay for Synovial IL-6 Levels}

Synovial fluids were diluted at least 1:1 with the assay diluent of respective ELISA kits, and the level of IL-6 (R\&D Systems, Minneapolis, MN, United States; D6050) was measured using the relevant ELISA kits ( $\& \& D$ Systems) according to the manufacturer's instructions.

\section{Quantitative RT-PCR}

HFLS, OA-FLS, and RA-FLS were seeded in six wells, and IL-6 was treated for $48 \mathrm{~h}$. Total RNA was isolated with RiboEX ${ }^{\mathrm{TM}}$ (GeneAll; 301-902). Isopropanol was mixed with isolated RNA on ice for $10 \mathrm{~min}$. All samples were centrifuged at $12,000 \mathrm{~g}, 4^{\circ} \mathrm{C}$. Supernatants were removed, and all samples were treated with $70 \%$ ethanol. All samples were centrifuged at 7,500 $\mathrm{g}$ for $10 \mathrm{~min}$ at $4^{\circ} \mathrm{C}$, and the pellets were air dried. RNA was dissolved in RNase-free water and quantitated using the spectrophotometer ND-1000 (Thermo Fisher Scientific, Waltham, MA, United States). Complementary DNA (cDNA) was amplified with Enzynomics (Daejeon, South Korea) following the manufacturer's instructions. Quantitative reverse transcription PCR (qRT-PCR) was performed using Power Up ${ }^{\mathrm{TM}}$ SYBRTM Green Master Mix (Applied Biosystems ${ }^{\circledR}$, Waltham, MA, United States; A25741), and the cycling protocol was as follows: human GAPDH (forward) GAC AGT CAG CCG CAT CTT CT and (reverse) TGA TGA CCC TTT TGG CTC CC; human NKCC1 (forward) GGA GTG GAA GAC CAC GTG AAA and (reverse) CCC ATA TGT ACA TGG CCA CAG A; human AQP-1 (forward) CAG TGA CCT CAC AGA CCG C and (reverse) TCT ATT TGG GCT TCA TCT CCA CC under UDG activation at $50^{\circ} \mathrm{C}$ for $2 \mathrm{~min}$, Dual-Lock DNA polymerase at $95^{\circ} \mathrm{C}$ for $2 \mathrm{~min}$, denaturation at $95^{\circ} \mathrm{C}$ for $15 \mathrm{~s}$, annealing at $59^{\circ} \mathrm{C}$ for $15 \mathrm{~s}$, and extension at $72^{\circ} \mathrm{C}$ for $1 \mathrm{~min}$.

\section{Osmolarity}

Synovial fluids were centrifuged at $1,500 \mathrm{rpm}$ for $15 \mathrm{~min}$ to remove cellular debris and liquid phase of supernatant. After heating for $10 \mathrm{~min}$ at $37^{\circ} \mathrm{C}$, the osmolarity of synovial fluids was measured from OA and RA samples using a Fiske ${ }^{\mathrm{TM}} 210$ Microsample Osmometer (Advanced Instruments, Inc., Norwood, MA, United States).

\section{Analysis of $\mathrm{Na}^{+}-\mathrm{K}^{+}-2 \mathrm{Cl}^{-}$ Co-transporter1 Activity}

$\mathrm{Na}^{+}-\mathrm{K}^{+}-2 \mathrm{Cl}^{-}$co-transporter1 (NKCC1) activity was measured based on the rate of $\mathrm{pH}_{i}$ decrease induced by intracellular $\mathrm{NH}_{4}{ }^{+}$

uptake (Evans and Turner, 1997) to determine the electrolyte influx for $\mathrm{Na}^{+}, \mathrm{K}^{+}$, and $\mathrm{Cl}^{-}$. To measure transporter activity, FLS cells were attached to a poly-L-lysine-coated coverslip and became adherent during culture using the perfusion-based technique. FLS were attached to coverslips for the measurement of NKCC1 activity. The administration of $20 \mathrm{mM} \mathrm{NH} 4 \mathrm{Cl}$ in the regular solution (composition is shown in Table 1) induced initial alkalization by $\mathrm{NH}_{3}$ diffusion; $\mathrm{pH}_{i}$ is then decreased due to $\mathrm{NH}_{4}^{+}$influx (pulse) as a substitute for $\mathrm{K}^{+}$. The $\mathrm{pH}_{i}$ recovery rate in the second phase following the $\mathrm{NH}_{4}{ }^{+}$pulse in the first alkalization phase was defined as the acidification rate $\left(\Delta \mathrm{pH}_{i} / \mathrm{s}\right)$. The slope of acidification rate by $\mathrm{pH}_{i}$ decrease was considered as the NKCC activity. The emitted fluorescence of the $\mathrm{pH}$ indicator BCECF-AM was monitored with a CCD camera (Teledyne Photometrics, Tucson, AZ, United States) attached to an inverted fluorescence microscope (Olympus, Tokyo, Japan) and was analyzed with a MetaFluor system (Molecular Devices, San Jose, CA, United States).

\section{Measurement of Cellular Volume Changes}

The FLS were loaded with the volume indicator calcein-AM ( $2 \mu \mathrm{M}$, Molecular Probes) in the presence of $0.01 \%$ pluronic acid (F-127) for $15 \mathrm{~min}$ at room temperature. After stabilization of fluorescence, the cells were perfused with HEPES-buffered solution for a minimum of $5 \mathrm{~min}$ prior to volume measurement. The calcein-AM was excited at $495 \mathrm{~nm}$, and the emitted fluorescence was measured at $515 \mathrm{~nm}$. The volume of FLS or HEK293T cells was determined using a hypertonic $(400 \mathrm{mOsm} / \mathrm{L}$, Table 1) solution (Hwang et al., 2019). Fluorescence images were automatically captured at 1-s intervals using a CCD camera (Q-Imaging, British Columbia, Canada, Retiga 6000) linked to an inverted microscope (Olympus, Tokyo, Japan) and analyzed with the MetaFluor system (Molecular Devices). Each signal was normalized by subtracting the background fluorescence signals to eliminate the raw background signals. RVI was followed by hypertonic stimulation-mediated cell shrinkage. The RVI rate of FLS was determined with slope of RVI increase and compared to that of the non-stimulated control group.

\section{DNA Transfection Procedure}

The human NKCC1 clone (pcDNA3.1) was provided by Dr. Shmuel Muallem at the National Institutes of

TABLE 1 | Composition of solution (mM).

Composition

Sodium chloride $(\mathrm{NaCl})$

HEPES

D-Glucose

Potassium chloride (KCl)

Magnesium chloride $\left(\mathrm{MgCl}_{2}\right)$

Calcium chloride $\left(\mathrm{CaCl}_{2}\right)$

$\mathrm{pH}$

Osmolarity
Regular solution Hypertonic-buffered solution

$\begin{array}{cc}140 & 140 \\ 10 & 10 \\ 10 & 10 \\ 5 & 5 \\ 1 & 1 \\ 1 & 1 \\ \mathrm{pH} 7.4 & \mathrm{pH} 7.4 \\ 00 \mathrm{mOsm} / \mathrm{L} & 400 \mathrm{mOsm} / \mathrm{L}\end{array}$


Health at Bethesda, MD, United States. Plasmid DNAs were incubated in $200 \mu \mathrm{l}$ of Jet prime buffer (Polyplus, Graffenstaden, France, \#B200225) and mixed with $4 \mu \mathrm{l}$ of the transfection reagent (Polyplus; \#21Y0910L1) for 10 min. DNAs were then transferred into HEK293Tcell plates containing culture medium, and all procedures were performed according to the manufacturer's protocol (Polyplus).

\section{Confocal Microscopy for Immunostaining}

The FLS were cultured on coverslips before being fixed with chilled methanol for $10 \mathrm{~min}\left(-20^{\circ} \mathrm{C}\right)$. Immunostaining was performed as previously described (Lee et al., 2016) using a 1:100 dilution of NKCC1 and AQP-1 antibodies. Briefly, the bound primary antibodies were detected with fluorescein isothiocyanate (FITC) or Rhodamine (1:100 dilution factor). Coverslips were mounted onto coated glass slides (SuperFrost $^{\text {plus }}$, Fisher) with DAPI-included fluoromount- $\mathrm{G}^{\mathrm{TM}}$ (Electron Microscopy Sciences, Hatfield, PA, United States), and images were analyzed using a LSM 700 Zeiss confocal microscope with ZEN software (Fluo-view, Carl Zeiss, Jena, Germany). Images were collected from five separate preparations of FLS, and results were presented as the average from all experiments.

\section{Western Blotting}

The FLS were pre-treated with IL-6 for $48 \mathrm{~h}$. Lysates of FLS were prepared in lysis buffer containing (mM) 20 Tris, $150 \mathrm{NaCl}, 2 \mathrm{EDTA}, 1 \%$ Triton X-100, and a protease inhibitor mixture and were treated as previously described (Lee et al., 2016). Briefly, cellular proteins were denatured in sodium dodecyl sulfate (SDS) sample buffer at $37^{\circ} \mathrm{C}$ for $30 \mathrm{~min}$. Denatured protein samples $(30 \mu \mathrm{g})$ were subjected to SDS polyacrylamide gel electrophoresis (SDSPAGE). Proteins were visualized with NKCC1, OSR-1, pOSR-1, AQP-1, and $\beta$-actin antibodies using enhanced luminescence solution (Thermo Fisher Scientific, Waltham, MA, United States) and developed on an X-ray film (Kodak, Tokyo, Japan).

\section{Statistical Analysis}

All experiments were repeated at least three times, and data were expressed as mean \pm standard error of the mean (SEM). Our study is based on the fact that IL-6 is one of the main inflammatory cytokines and hypothesize these inflammatory circumstances of RA influence on cellular osmotic modules. To verify this, significant difference of IL-6 between RA and OA was needed. Thus, the sample size was calculated by power $80 \%$ and significance $5 \%$ (two-sided) on the basis of the article that showed the difference of synovial fluid IL-6 levels between patients with RA and OA (Okamoto et al., 1997). Statistical differences between the mean values from the OA and RA synovial fluid groups and samples were analyzed using Student's $t$-tests. Statistical significance was defined as $\mathrm{p}\left({ }^{*} p<0.05\right.$, $\left.* * p<0.01,{ }^{* * *} p<0.001\right)$.

\section{RESULTS}

\section{Synovial Fluid IL-6 Level Was Enhanced in RA Patients Compared to OA Patients}

Synovial fluids were obtained from 16 subjects, 8 with RA and 8 with OA (Okamoto et al., 1997). The demographic data of the subjects, including age, gender, and disease duration on the day before surgery, are summarized in Figure 1A. The mean age in the RA group was 64 years; in the OA group, it was 70 years. All of the patients with RA were on the combination therapy with more than one conventional synthetic DMARDs and nonsteroidal anti-inflammatory drugs (NSAIDs). The patients with OA were taking NSAIDs or acetaminophen. We determined the osmolarity and the content of IL- 6 in synovial fluid to verify the role of IL-6 in cytotoxic edema. The gender of the subjects was not considered. The synovial osmolarity of RA patients was mildly enhanced compared to that of OA patients; however, there was no statistical difference (Figure 1B). The synovial IL-6 levels were enhanced in RA patients compared to OA patients, in agreement with other previous studies (Figure 1C; Kokebie et al., 2011; Machado Diaz et al., 2012; Hampel et al., 2013; Elicabe et al., 2017). There was no statistical difference in represented age of patients between the RA and OA groups (Figures 1B,C).

\section{IL-6-Stimulated RA-FLS Were Sensitive to Hypertonic Stimulation}

We examined IL-6-mediated osmotic regulation in FLS. The experiment was performed using hyperosmotic stimulation, and the RVI rate was measured. IL-6-stimulated RA-FLS displayed enhanced RVI (Figures 2A,B). RVIs of HFLS and OA-FLS were not statistically different between control and IL-6-stimulated groups (Figures 2C-F). RA synovial fluid contained IL-6 as shown in Figure 1C. Thus, we measured RVI of RA-FLS in the presence of RA synovial fluid. RA synovial fluid-pre-treated RAFLS enhanced RVI by hypertonic stimulation (Figures $2 \mathbf{G}, \mathbf{H}$ ). These results demonstrate that IL-6-stimulated RA-FLS were more sensitive to hypertonic stimulation than either HFLS or OA-FLS, and RA synovial fluid stimulated enhanced osmotic sensitivity of RA-FLS.

\section{IL-6 Enhanced NKCC1 Activity in HFLS and RA-FLS}

RVI caused by osmotic cell swelling requires the involvement of NKCC1 (Hoffmann and Dunham, 1995). Here, we examined the NKCC1 protein expression in IL-6-treated FLS. There were no differences in the messenger RNA (mRNA) and protein expression of NKCC1 of three types of FLS based on IL-6 treatment (Figures $\mathbf{3 A}-\mathbf{C}$ ). To evaluate the role of NKCC1 in volume change, HEK293T cells were made to overexpress NKCC1. Hypertonic stimulation induced a 10-fold higher volume shrinkage in NKCC1-overexpressed HEK293T cells when compared to the wild type of HEK293T cells (control) (Figure 3D). Although the expression level of NKCC1 did not change in both control (HFLS) and RA-FLS based on treatment with IL-6, we determined the NKCC1 activity in the presence of IL-6. NKCC1 activity in HFLS and RA-FLS was enhanced by 
A

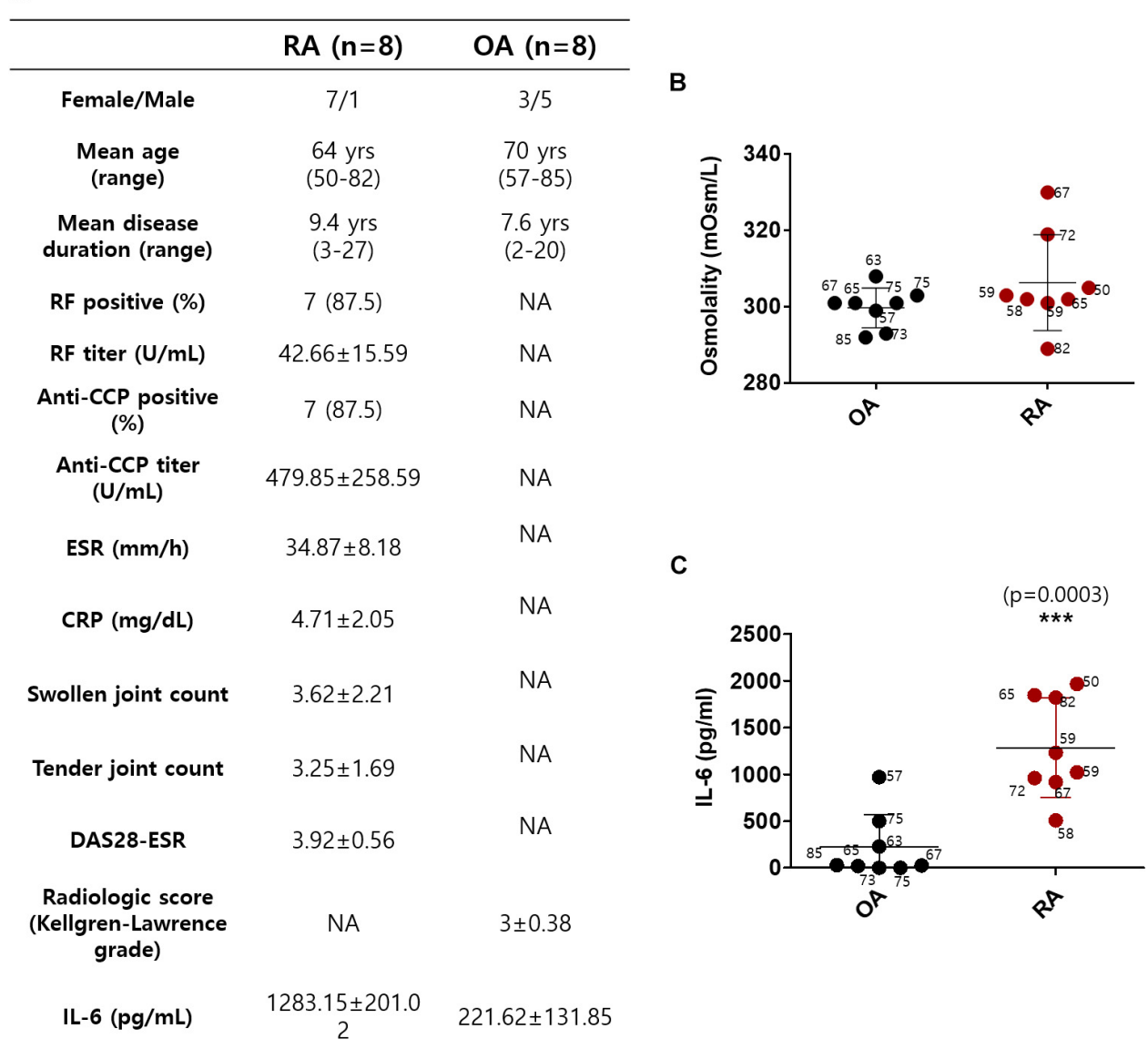

FIGURE 1 | Synovial fluid interleukin (IL)-6 level was enhanced in rheumatoid arthritis (RA) patients compared to osteoarthritis (OA) patients. (A) Demographic data of RA patients and OA patients. (B) Osmolarity (mOsm/L) of synovial fluids in OA and RA patients. (C) Measurement of IL-6 levels in synovial fluids of OA and RA patients using ELISA assay $\left(n=8,{ }^{\star \star *} p<0.001\right.$, ${ }^{*}$ : OA vs RA).

treatment with IL-6, whereas NKCC1 activity of OA-FLS was mildly enhanced with no statistical difference (Figures $3 \mathbf{E}-\mathbf{H}$ ). NKCC1 activity of RA-FLS was highly responsive to the IL-6 treatment compared with HFLS and OA-FLS.

\section{IL-6 Stimulated Membranous Expression of NKCC1 and AQP-1}

NKCC1 recruits water channels to maintain volume homeostasis. Among those water channels, AQP-1 was expressed in FLS and revealed enhanced expression in RA (Trujillo et al., 2004; Mu et al., 2020). We confirmed that treatment with IL-6 enhanced mRNA and protein expression of AQP-1 in RA-FLS (Figures 4AC). Additionally, NKCC1 and AQP-1 were stained with and without IL-6 treatment. We found recruitment of NKCC1 on the plasma membrane in IL-6-treated RA-FLS (Figure 4D). We measured the region of interest of the membrane and cytosolic area. Nuclear immunoreactivity of AQP-1 was also observed. Membranous expression of NKCC1 and AQP-1 was enhanced in RA-FLS (Figures 4D,E), whereas those of HFLS and OAFLS had no difference by IL-6 stimulation (Figures 4F-I and Supplementary Material). No changes in nuclear staining of
AQP-1 were observed in the three types of FLS (Figures 4E,G,I). Cellular volume and osmotic regulation were mediated by the involvement of OSR-1 (Sengupta et al., 2013). Enhanced hypertonic sensitivity and osmotic regulation by IL-6 may induce the phosphorylation of OSR-1. Thus, we determined the protein expression of OSR-1 in the presence of IL-6. The phosphorylated and total forms of OSR-1 showed no change with IL- 6 treatment after $48 \mathrm{~h}$ (Figures 4J,K). These results demonstrate that enhanced NKCC1 and AQP-1 expression was a complementary process working against osmotic changes caused by IL-6stimulated ion flux; these mechanisms operated without changing the expression of the osmolarity-sensitive protein OSR-1.

\section{RA Synovial Fluid Enhanced NKCC1 Activity, and IL-6 Antibody Blocked the Effect of RA Synovial Fluid in RA-FLS}

The concentration of IL-6 in RA synovial fluid was 6-fold higher than that of OA synovial fluid as shown in Figure 1C. We determined whether the synovial fluid of RA patients directly enhanced NKCC1 activity. Direct stimulation with synovial fluid on FLS was technically limited due to its viscosity and 


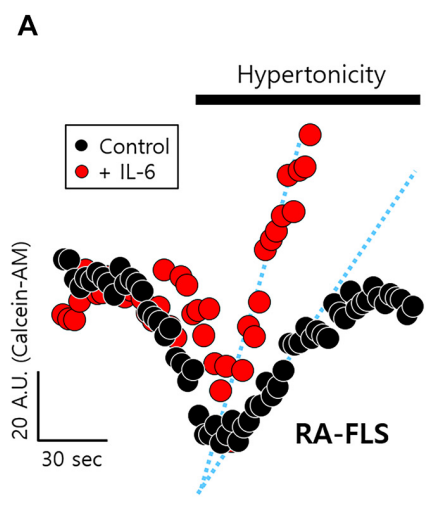

B

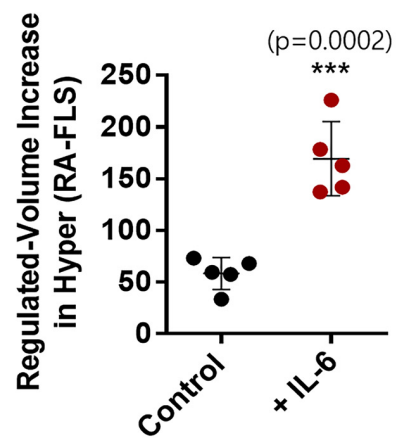

C

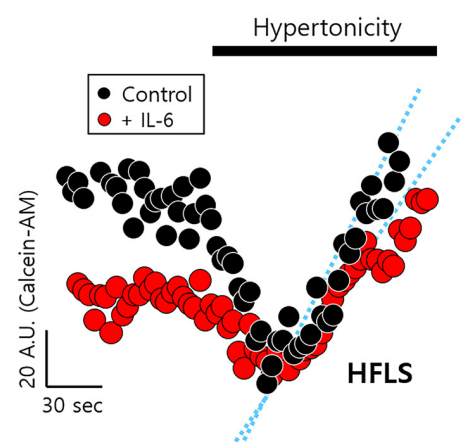

D

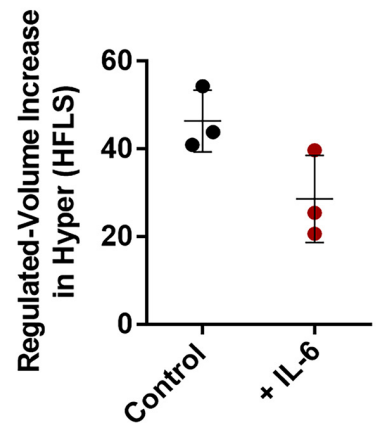

E

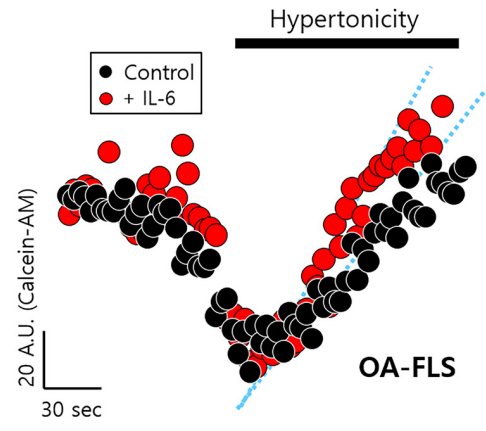

F

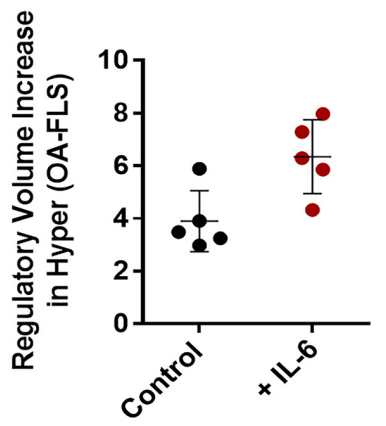

G

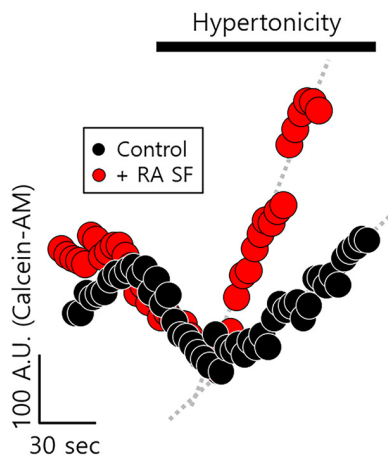

H

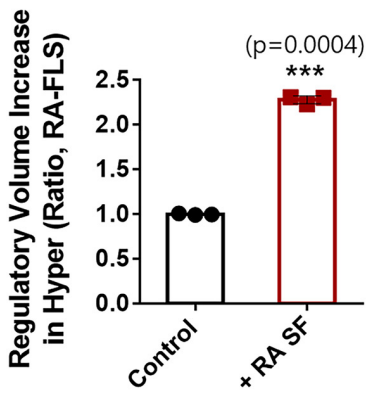

FIGURE 2 | Interleukin (IL)-6-stimulated rheumatoid arthritis fibroblast-like synoviocytes (RA-FLS) were sensitive to hypertonic stimulation. Volume changes in FLS on stimulation with a hypertonic solution (400 mOsm/L) pre-treated with or without $50 \mathrm{ng} / \mathrm{ml} \mathrm{IL-6} \mathrm{for} 48 \mathrm{~h}$ in (A) RA-FLS, (C) human FLS (HFLS), and (E) osteoarthritis FLS (OA-FLS). The bars represent the mean \pm SEM of volume changes with or without IL-6 treatment in (B) RA-FLS, (D) HFLS, and (F) OA-FLS. [** $P<0.001,{ }^{*}$ : Control vs + (IL)-6]. (G) Volume changes in RA-FLS on stimulation with a hypertonic solution (400 mOsm/L) pre-treated with or without synovial fluid of RA patients for $6 \mathrm{~h}$ in a migration assay. $(\mathbf{H})$ The bars represent the mean \pm SEM of volume changes with or without synovial fluid of RA patients in RA-FLS. Hypertonic solution (hyper) $\left(n=3,{ }^{\star \star *} p<0.001,{ }^{*}\right.$ : Control vs + RA SF).

turbidity. Thus, RA-FLS were stimulated with synovial fluid in the bottom chamber of the Boyden Transwell system to prevent these obstacles to imaging NKCC1 activity. Three synovial fluids of RA and OA were randomly selected, respectively. Stimulation with RA synovial fluid enhanced NKCC1 activity in RA-FLS (Figures 5A,C), whereas OA synovial fluid revealed no differences between the control and stimulated group (Figures 5B,D). In addition, we hypothesized that RA synovial fluids, including IL-6, activated FLS. To remove the effect of IL-6 in RA synovial fluid, IL-6 antibody was pre-treated in RA synovial fluid. Enhanced NKCC activity and RVI by RA synovial fluid were diminished by the pretreatment of IL- 6 antibody in RA synovial fluid (Figures 5E-H). These results confirmed that RA synovial fluid enhanced NKCC1 activity, and the treatment of IL-6 antibody blocked the effects of RA synovial fluid on the modulation of NKCC activity and RVI in RA-FLS. 

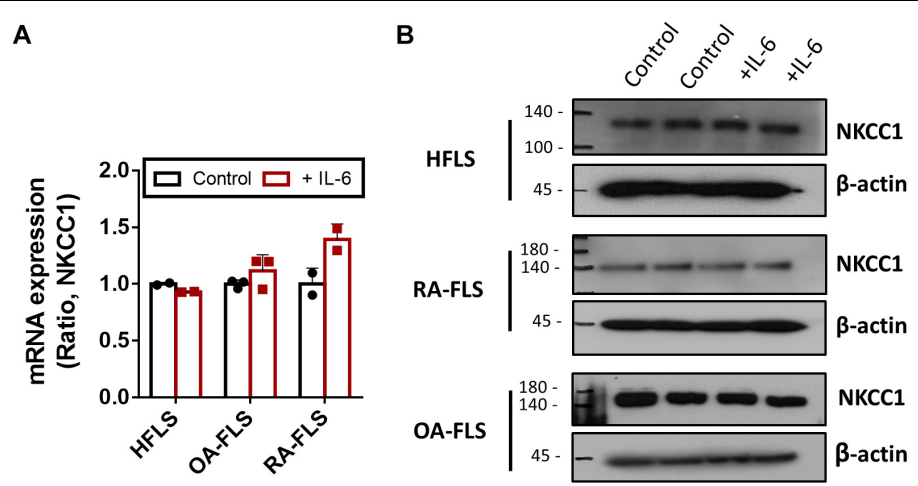

C

E

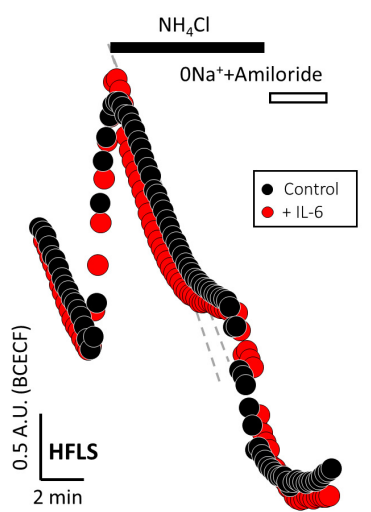

$\mathbf{F}$
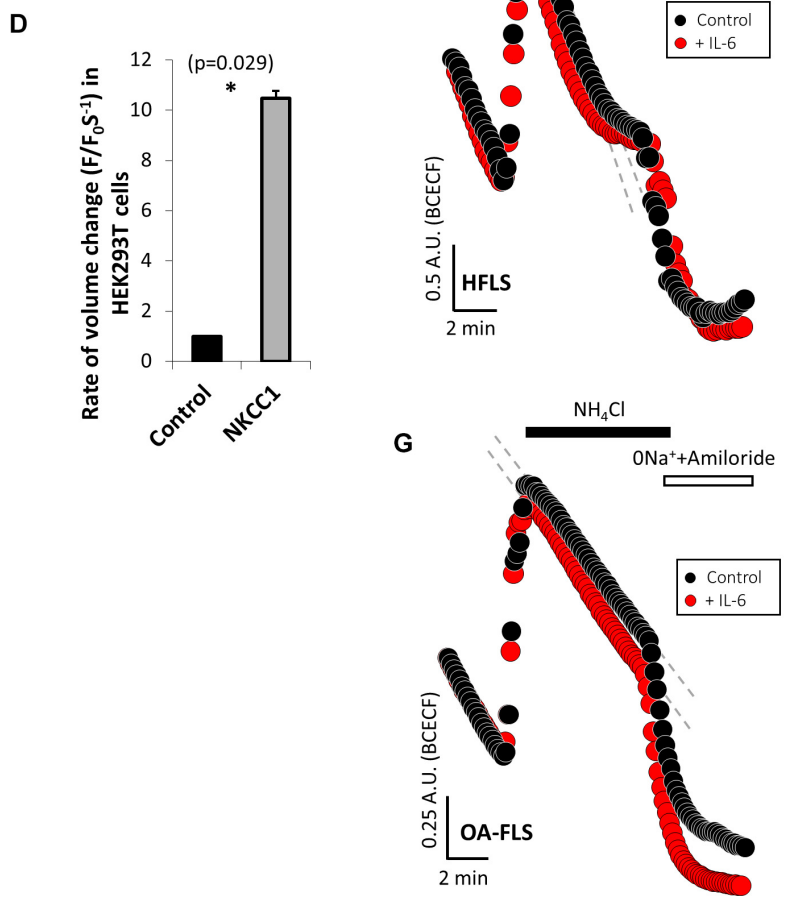

H
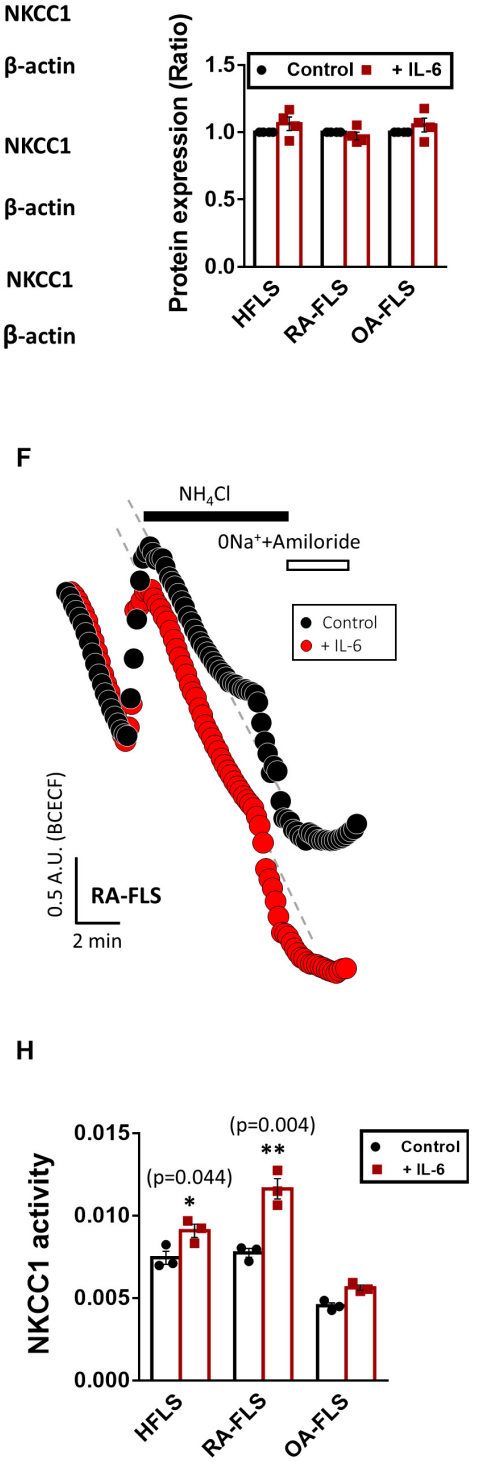

FIGURE 3 | Interleukin (IL)-6 enhanced sodium-potassium-chloride cotransporter 1 (NKCC1) activity in human fibroblast-like synoviocytes (HFLS) and rheumatoid arthritis FLS (RA-FLS). (A) mRNA levels and (B) protein levels of NKCC1 with pretreatment of $50 \mathrm{ng} / \mathrm{ml} \mathrm{IL-6}$ for $48 \mathrm{~h}$ in HFLS, OA-FLS, and RA-FLS. $\beta$-Actin was used as a loading control. (C) Analysis of band intensity of NKCC1 protein levels in HFLS, OA-FLS, and RA-FLS. (D) Rate of volume change by hypertonic stimulation in NKCC1-overexpressed HEK293T cells. Results are shown as mean \pm SEM $\left(n=3,{ }^{*} p<0.05\right.$, ${ }^{*}$ : Control vs NKCC1). NKCC1 activity due to the 20 mM $\mathrm{NH}_{4} \mathrm{Cl}$ pulse was measured with pretreatment of $50 \mathrm{ng} / \mathrm{ml} \mathrm{IL-6} \mathrm{in} \mathrm{(E)} \mathrm{HFLS,} \mathrm{(F)} \mathrm{RA-FLS,} \mathrm{and} \mathrm{(G)} \mathrm{OA-FLS.} \mathrm{(H)} \mathrm{Analysis} \mathrm{of} \mathrm{NKCC1} \mathrm{activity.} \mathrm{NKCC1} \mathrm{activity} \mathrm{of} \mathrm{the}$ IL-6-stimulated groups were normalized to control. Results are shown as mean \pm SEM $\left[n=3,{ }^{*} p<0.05,{ }^{* \star} p<0.01\right.$, ${ }^{*}$ : Control vs + (IL) -6$]$.

\section{DISCUSSION}

This study addressed for the first time whether RA synovial fluids or IL-6 would enhance the protein expression and activity of NKCC1 and complementary AQP-1 in RA-FLS. Osmotic changes in synovial fluids, such as the mildly hypertonic condition caused by RA, revealed more sensitive volume changes in RA-FLS than in OA-FLS or HFLS. Our study provided the pathophysiological role of NKCC1 and AQP-1 as inflammation-linked joint edema modules in the pathogenesis of RA patients.

Joint swelling is considered to be one of the 2010 ACREULAR classification criteria for RA diagnosis (Aletaha et al., 2010). We hypothesized that the osmotic change of synovium is associated with molecular edema module, such as NKCC1 or OSR-1 in FLS. RVI caused by hyperosmotic stimulation is mediated by ion influx with accompanying water and 
A
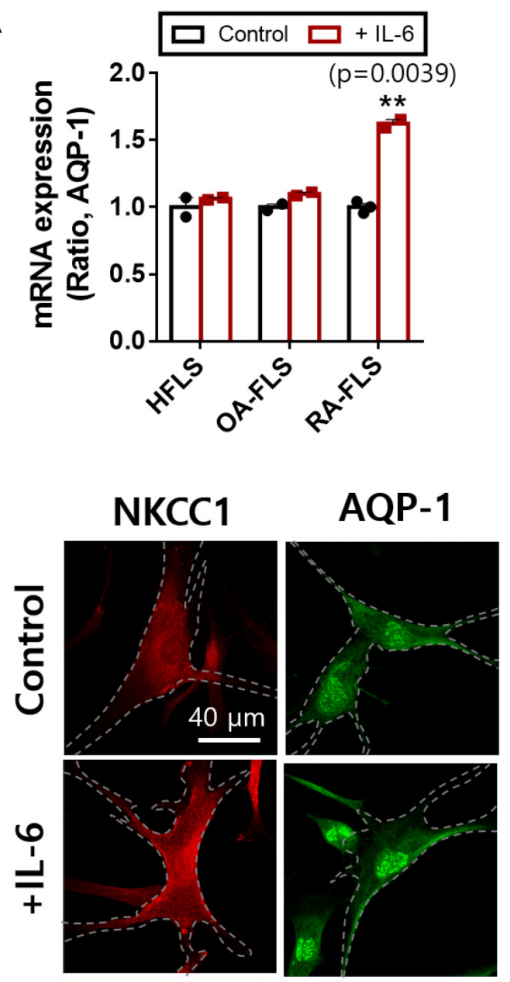

E

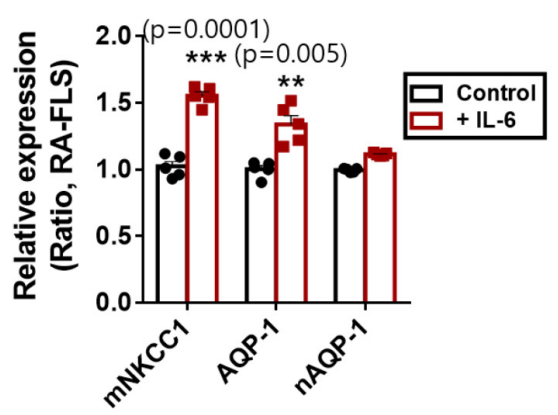

B

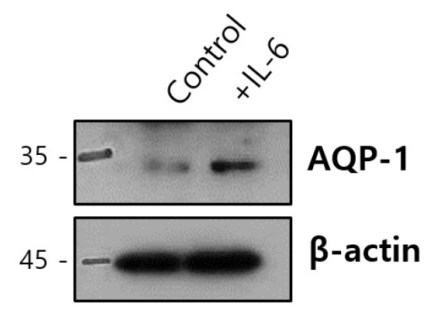

C

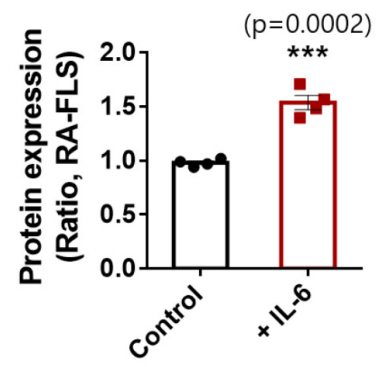

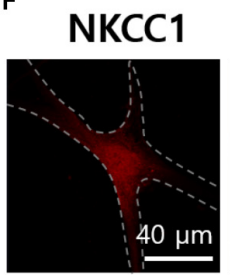

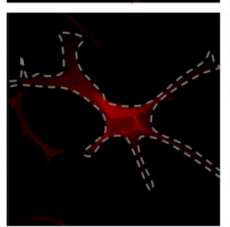

G

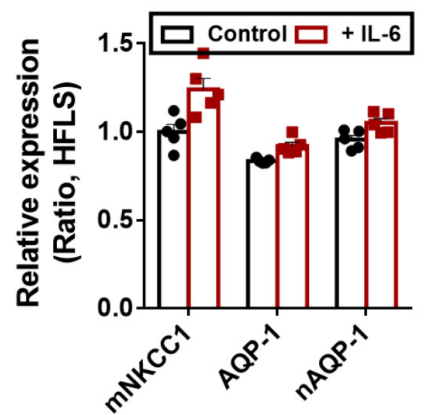

H

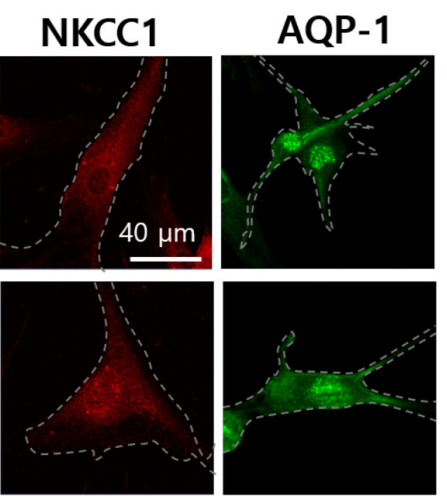

I

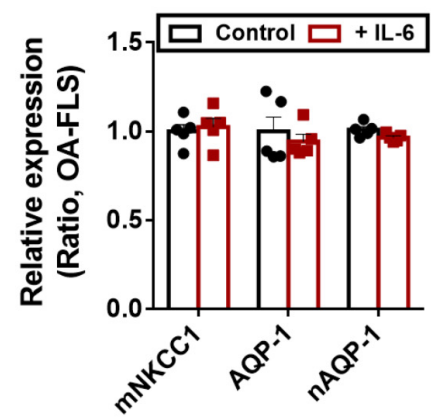

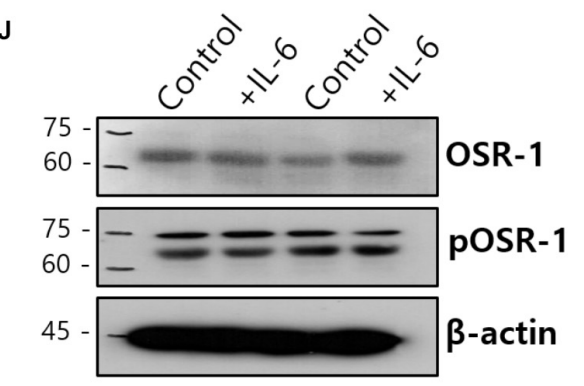

K

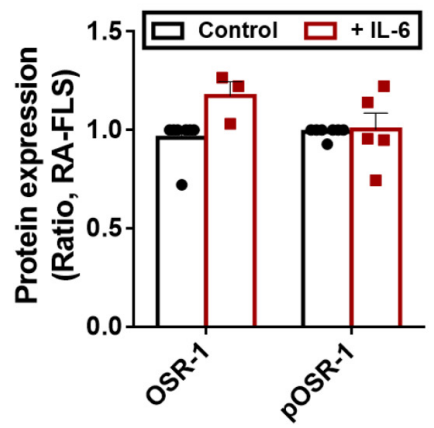

FIGURE 4 | Interleukin (IL)-6 enhanced aquaporin (AQP)-1 expression in rheumatoid arthritis fibroblast-like synoviocytes (RA-FLS). (A) mRNA levels [ $n=3$, ${ }^{* *} \mathrm{P}<0.01$, *: Control vs $\left.+(\mathrm{IL})-6\right]$ and (B) protein levels of AQP-1 with pretreatment of $50 \mathrm{ng} / \mathrm{ml} \mathrm{lL}-6$ for $48 \mathrm{~h}$ in human FLS (HFLS), osteoarthritis FLS (OA-FLS), and RA-FLS. $\beta$-Actin was used as a loading control. (C) Analysis of band intensity of AQP-1 protein levels in RA-FLS $\left[n=4,{ }^{* \star *} p<0.001,{ }^{*}\right.$ : Control vs $+($ (IL) -6$]$. Immunolocalization of NKCC1 (red) and AQP-1 (green) with treatment of $50 \mathrm{ng} / \mathrm{ml} \mathrm{IL-6} \mathrm{in} \mathrm{(D)} \mathrm{RA-FLS,} \mathrm{(F)} \mathrm{HFLS,} \mathrm{and} \mathrm{(H)} \mathrm{OA-FLS.} \mathrm{Analysis} \mathrm{of} \mathrm{membranous} \mathrm{intensity}$ of sodium-potassium-chloride cotransporter1 (NKCC1) and AQP-1 and nuclear staining of AQP-1 in (E) RA-FLS $\left[n=5,{ }^{* *} p<0.01\right.$ and ${ }^{* * *} p<0.001,{ }^{*}$ : Control vs + (IL)-6], (G) HFLS, and (I) OA-FLS. (J) Protein levels of OSR-1 and pOSR-1 with treatment of $50 \mathrm{ng} / \mathrm{ml}$ IL-6 for $48 \mathrm{~h}$ of RA-FLS. $\beta$-Actin was used as a loading control. (K) Analysis of band intensity of OSR-1 and pOSR-1 in RA-FLS. 
A

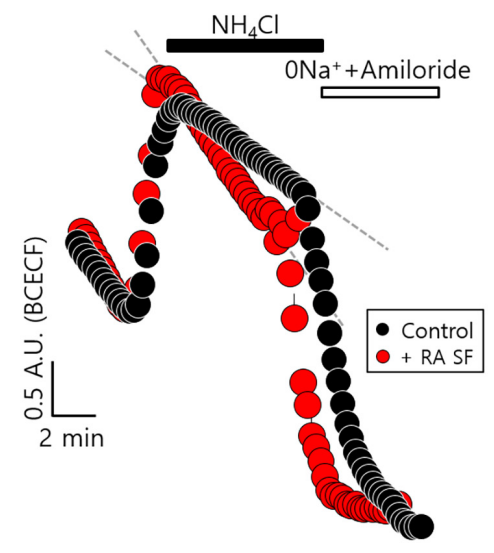

C

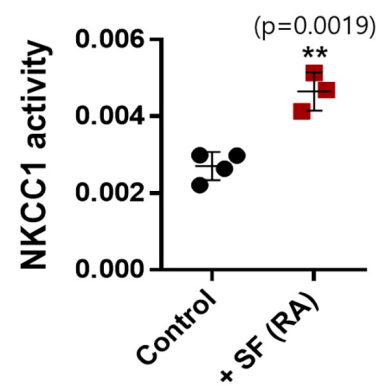

E

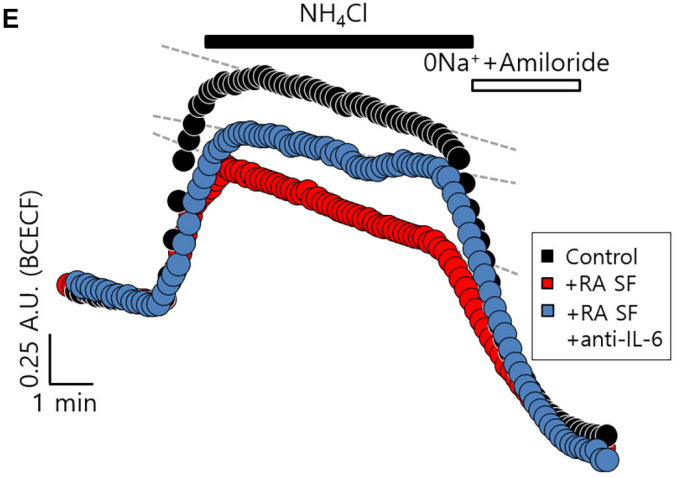

G

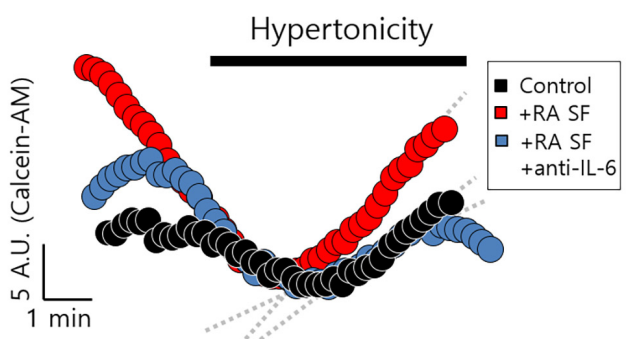

B

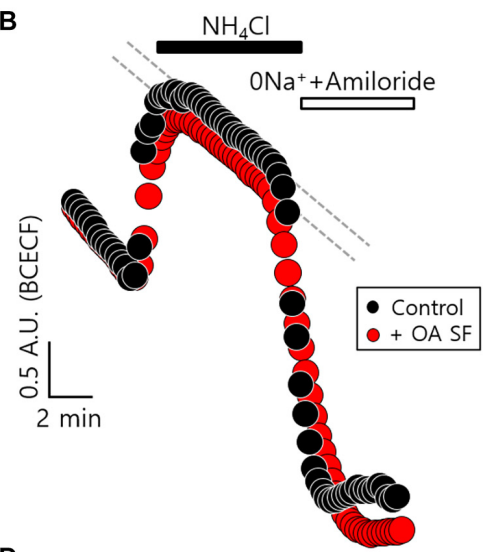

D

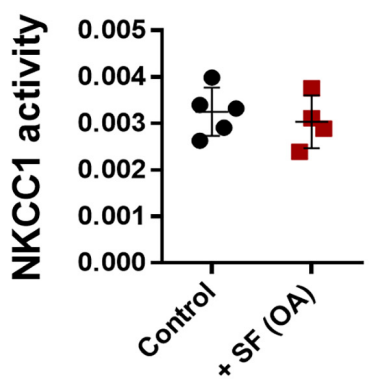

F

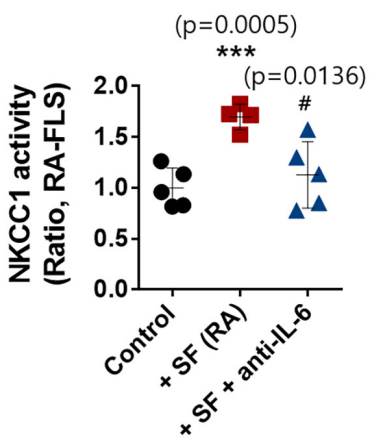

H

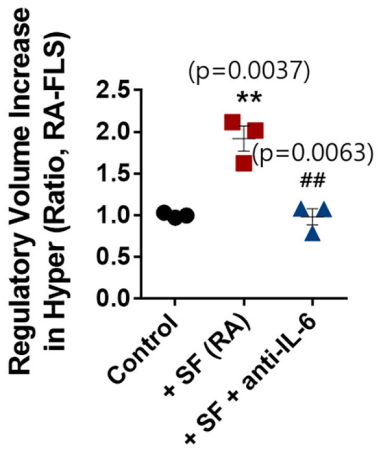

FIGURE 5 | Rheumatoid arthritis (RA) synovial fluid enhanced sodium-potassium-chloride cotransporter1 (NKCC1) activity and interleukin (IL)-6 antibody blocked the effect of RA synovial fluid in RA fibroblast-like synoviocytes (RA-FLS). NKCC1 activity was measured using the membrane of a Boyden Transwell chamber with pretreatment with (A) RA and (B) osteoarthritis (OA) synovial fluid for $6 \mathrm{~h}$ in RA-FLS. The bars represent the mean \pm SEM of NKCC1 activity with or without (C) RA $\left[n=4,{ }^{*} p<0.01,{ }^{*}\right.$ : Control vS + SF (RA)] and (D) OA synovial fluid in RA-FLS. (E) NKCC1 activity by the stimulation of RA synovial fluid with or without $5 \mu \mathrm{g} / \mathrm{ml}$ anti-IL- 6 antibody for $6 \mathrm{~h}$ in RA-FLS on the membrane of Boyden Transwell chamber. (F) The bars represent the mean \pm SEM of NKCC1 activity $[n=5$,

${ }^{\star \star *} p<0.001$, and $\# p<0.05$, *: Control vs + SF (RA), \#: + SF (RA) vs + SF + anti-(IL)-6]. (G) Volume changes in RA-FLS on stimulation with a hypertonic solution (400 mOsm/L). RA synovial fluid was pre-treated with $5 \mu \mathrm{g} / \mathrm{ml}$ anti-IL-6 antibody for $1 \mathrm{~h}$ and then stimulated to RA-FLS on the membrane of Boyden transwell chamber for 6 h. (H) The bars represent the mean \pm SEM of volume changes. Hypertonic solution (hyper) $\left[n=3,{ }^{* *} p<0.01\right.$ and ${ }^{\# \#} p<0.01$, ${ }^{*}$ : Control vs + SF (RA), \#: + SF (RA) vs + SF + anti-(IL)-6]. 


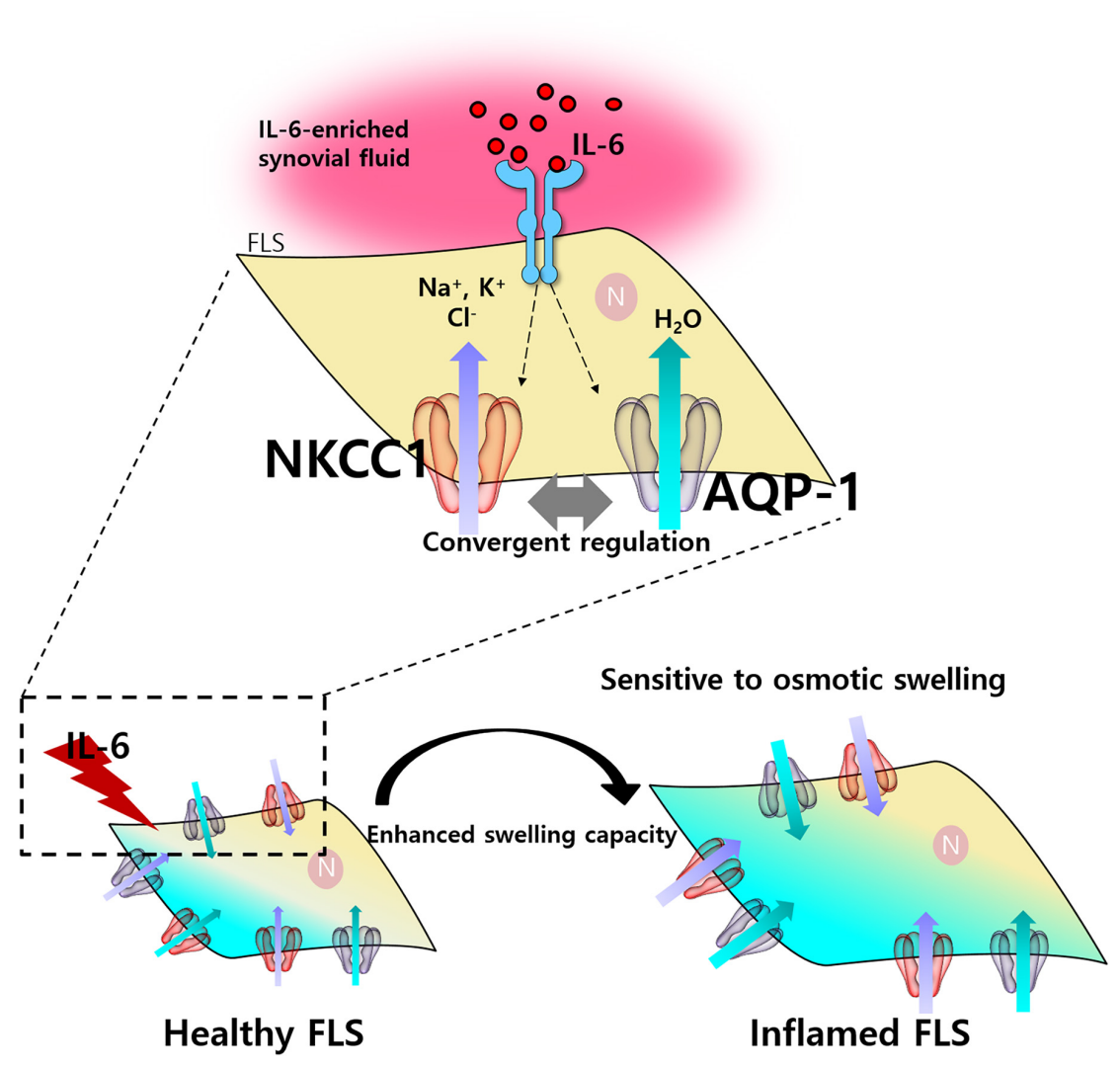

FIGURE 6 | Schematic illustration of osmotic change by interleukin (IL)-6 through sodium-potassium-chloride cotransporter1 (NKCC1) and aquaporin (AQP)-1 in fibroblast-like synoviocytes (FLS). IL-6-induced activation of NKCC1 and AQP-1 in FLS. Activation of NKCC1 and AQP-1 by IL-6/IL-6-enriched synovial fluid resulted in the influx of solute and water causing the sensitivity to hyperosmotic stimulation.

phosphorylation of NKCC1 and activation of OSR-1 (Alessi et al., 2014). Our results suggest that RA-FLS possessed high potential of RVI through the convergent regulation of NKCC1 and AQP-1 in response to proinflammatory cytokines within synovial fluid, including IL-6. It has been suggested that levels of IL-6 are associated with cytotoxic edema (Takano et al., 2002).

RA synovial fluids were observed to have a relatively high cytokine level compared to OA samples in Figure 1A. Many reports used RA/OA comparison based on current knowledge as shown in Plebanczyk et al. (2019). For comparison between healthy control and RA patient, it is difficult to obtain synovial fluid from healthy persons because it cannot be aspirated sufficiently for our experiment (at least more than $10 \mathrm{ml}$ ). Related to the joints from healthy persons who have surgery due to trauma, they actually did not mean complete healthy tissues because they have complex healing process including inflammation, although enough synovial fluid could be collected. Several inflammatory cytokines including IL-6 have been found in synovial fluids from RA patients. The profile of several inflammatory cytokines, such as IL-1, IL6, IL-8, IL-18, and IL-29 in synovial fluids was associated with RA (Kaneko et al., 2000; Shao et al., 2009; Kokebie et al., 2011; Wang et al., 2012). The role of IL-6 on RA was clearly shown in IL-6-mediated osmotic regulation and enhanced NKCC activity in RA-FLS and concentration of IL-6 in RA synovial fluids. The administration of IL6 antibody on RA synovial fluids also revealed the reduced osmotic responsiveness and NKCC1 activity in RA-FLS as shown in Figures 5E-H. However, therapeutic approach for the blockade of IL-6 or IL-6 receptor should require careful consideration. The IL-6 receptor blocker, tocilizumab, has enhanced serum IL-6 level (Nishimoto et al., 2008). Tocilizumab-associated enhanced IL-6 level may enhance cytotoxic edema module as addressed by our results. Thus, Figure 6 represents a schematic illustration of this osmotic regulation mechanism in RA-FLS.

Additionally, we explored the mechanisms of osmotic regulation in inflammatory mediator-induced cytotoxic edema, which also occurred in the joint area. Generally, edema is classified in the cerebral region and divided as vasogenic, cytotoxic, or interstitial edema (Bhardwaj, 2007). We hypothesized, as mentioned in the beginning of this paragraph, that FLS could also be elements in cytotoxic edema just as neurons and endothelial cells are in the cerebral regions. In this study, we proposed the concept of synovial ischemia to represent the swelling of FLS caused by the influx of electrolytes and water similar to that seen in cerebral ischemia (Dijkstra et al., 2016). The current study reveals that AQP-1 is clearly implicated 
in the development of joint edema caused by FLS swelling in the inflammatory synovium, similar to AQP-4 mediated astrocyte swelling in brain edema (Rao et al., 2011). In addition, ischemic heart injury is mediated by the involvement of NKCC1 (Ramasamy et al., 2001). IL-6 activates NKCC1 activity in cardiac tissue (Ji et al., 2020) and induces NKCC1 phosphorylation and $\mathrm{Cl}^{-}$accumulation in sensory neurons (Pieraut et al., 2011). The enrollment of NKCC1 is also associated with cellular volume change, membrane permeability, and subsequent change in water content in the brain (Gong et al., 2021). Additionally, the multiple roles of IL- 6 spread throughout both inflammatory and metabolic processes, making contributions to both of these vital pathways (Jones and Jenkins, 2018). The signaling axis of IL-6 is considered the central process of inflammation and autoimmunity in various tissues including pulmonary, cardiac, and joints (Hirano et al., 1987; Tanaka et al., 2014; Ji et al., 2020; Wang and He, 2020; Gong et al., 2021). IL-6 induces enhanced production of vascular endothelial growth factor, resulting in enhanced angiogenesis and vascular permeability (Nakahara et al., 2003) and induced inflammatory signal of immune cells including $\mathrm{T}$ helper 17 (Th17) cells (Swaak et al., 1988; Bettelli et al., 2006; Yue et al., 2010). Th17 activation and arthritis activity, such as joint swelling of CIA mice were inhibited by the treatment of anti-IL-6R antibody (Fujimoto et al., 2008). In addition, joint inflammation was suppressed in IL-6-deficient mice (Nowell et al., 2003). In this study, our experimental evidence directly provide that the enhanced IL-6 level of RA patients compared to that of OA can be a switch to turn on the intracellular IL-6 signaling, such as enhanced cytotoxic edema modules NKCC1 and AQP-1 in RA. Although there are various degrees, these results implicate the pathophysiological role of inflamed synovial fluids in the pathogenesis of RA.

We expected the enhanced expression of NKCC1 in the presence of IL-6 or IL-6-enriched synovial fluid; however, the mRNA and protein expression was not changed. Membrane recruitment of transporters and channels was functionally working without increased net protein expression. In addition, RVI or RVD occurs in response to osmotic changes (O'Brien et al., 1993). We hypothesized that if osmolarity-sensitive proteins, such as NKCC1 are recruited to the plasma membrane, our experimental stimulation of hyperosmolar solution mediated cellular volume change. Our experiment was performed in a relatively high osmolarity of $400 \mathrm{mOsm} / \mathrm{L}$. We suggest that these data did not mean that synovial fluids were hyperosmolar. The RVI tool was used in the current study to verify the volume regulation of osmotic-responsive protein, such as NKCC1. Our results suggest that enhanced membrane recruitment of NKCC1 and combined water channels provide favorable circumstances for the homeostatic response to hyperosmotic stimulation in patients with inflamed synovial fluid. We propose that blocking the osmotic sensitivity of FLS may represent a new therapeutic

\section{REFERENCES}

Alessi, D. R., Zhang, J., Khanna, A., Hochdorfer, T., Shang, Y., and Kahle, K. T. (2014). The WNK-SPAK/OSR1 pathway: master regulator strategy not only for cytotoxic edema in RA but also for other osmotic dysfunctions including brain and cardiac edemas.

\section{DATA AVAILABILITY STATEMENT}

The raw data supporting the conclusions of this article will be made available by the authors, without undue reservation.

\section{ETHICS STATEMENT}

This study was conducted in accordance with the Declaration of Helsinki and was approved by the Institutional Review Board of Gil Medical Center (GAIRB-2019-018). Written informed consent was obtained from each individual.

\section{AUTHOR CONTRIBUTIONS}

$\mathrm{JH}$ and $\mathrm{MJ}$ contributed to the conception and design, acquisition, or analysis and interpretation of data. MJ and HR made the article drafts, acquired the data, and performed critical revisions for the important intellectual content. HR collected the synovial fluid from the human subjects. JH drew all schematics. JH and HR contributed to the final approval of the version to be published and agreed to be held accountable for all aspects of the work, ensuring that questions related to the accuracy or integrity of any part of the work are appropriately investigated and resolved. All authors contributed to the article and approved the submitted version.

\section{FUNDING}

This work was supported by the Gachon University research fund of 2020 (GCU-202002570001) and the grant from the National Research Foundation of Korea (NRF) grant funded by the Korean Government (MSIT) (NRF-2019R1F1A1046785).

\section{ACKNOWLEDGMENTS}

We thank Gachon University and Gil Medical Center for technical and funding support.

\section{SUPPLEMENTARY MATERIAL}

The Supplementary Material for this article can be found online at: https://www.frontiersin.org/articles/10.3389/fcell.2021. 700879/full\#supplementary-material

of cation-chloride cotransporters. Sci Sig. 7:re3. doi: 10.1126/scisignal. 2005365

Aletaha, D., Neogi, T., Silman, A. J., Funovits, J., Felson, D. T., Bingham, C.O. 3rd, et al. (2010). 2010 Rheumatoid arthritis classification criteria: an american 
college of rheumatology/european league against rheumatism collaborative initiative. Arthritis Rheum. 62, 2569-2581.

Altobelli, E., Angeletti, P. M., Piccolo, D., and De Angelis, R. (2017). Synovial fluid and serum concentrations of inflammatory markers in rheumatoid arthritis, psoriatic arthritis and osteoarthitis: a systematic review. Curr. Rheumatol. Rev. $13,170-179$

Bettelli, E., Carrier, Y., Gao, W., Korn, T., Strom, T. B., Oukka, M., et al. (2006). Reciprocal developmental pathways for the generation of pathogenic effector TH17 and regulatory T cells. Nature 441, 235-238. doi: 10.1038/nature04753

Bhardwaj, A. (2007). Osmotherapy in neurocritical care. Curr. Neurol. Neurosci. Rep. 7, 513-521. doi: 10.1007/s11910-007-0079-2

Deon, D., Ahmed, S., Tai, K., Scaletta, N., Herrero, C., Lee, I. H., et al. (2001). Crosstalk between IL-1 and IL-6 signaling pathways in rheumatoid arthritis synovial fibroblasts. J. Immunol. 167, 5395-5403. doi: 10.4049/jimmunol.167.9.5395

Dijkstra, K., Hofmeijer, J., Van Gils, S. A., and Van Putten, M. J. (2016). A biophysical model for cytotoxic cell swelling. J. Neurosci. 36, 11881-11890. doi: 10.1523/jneurosci.1934-16.2016

Elicabe, R. J., Silva, J. E., Dave, M. N., Lacoste, M. G., Tamashiro, H., Blas, R., et al. (2017). Association between IL-17 and IgA in the joints of patients with inflammatory arthropathies. BMC Immunol. 18:8. doi: 10.1186/s12865-0170189-9

Evans, R. L., and Turner, R. J. (1997). Upregulation of $\mathrm{Na}(+)-\mathrm{K}(+)-2 \mathrm{Cl}-$ cotransporter activity in rat parotid acinar cells by muscarinic stimulation. J. Physiol. 499, 351-359. doi: 10.1113/jphysiol.1997.sp021932

Fujimoto, M., Serada, S., Mihara, M., Uchiyama, Y., Yoshida, H., Koike, N., et al. (2008). Interleukin-6 blockade suppresses autoimmune arthritis in mice by the inhibition of inflammatory Th17 responses. Arthritis Rheum. 58, 3710-3719. doi: 10.1002/art.24126

Georganas, C., Liu, H., Perlman, H., Hoffmann, A., Thimmapaya, B., and Pope, R. M. (2000). Regulation of IL-6 and IL-8 expression in rheumatoid arthritis synovial fibroblasts: the dominant role for NF-kappa B but not C/EBP beta or c-Jun. J. Immunol 165, 7199-7206. doi: 10.4049/jimmunol.165.12.7199

Gong, Y., Wu, M., Shen, J., Tang, J., Li, J., Xu, J., et al. (2021). Inhibition of the NKCC1/NF-kappaB signaling pathway decreases inflammation and improves brain edema and nerve cell apoptosis in an sbi rat model. Front. Mol. Neurosci. 14:641993. doi: 10.3389/fnmol.2021.641993

Hampel, U., Sesselmann, S., Iserovich, P., Sel, S., Paulsen, F., and Sack, R. (2013). Chemokine and cytokine levels in osteoarthritis and rheumatoid arthritis synovial fluid. J. Immunol. Methods 396, 134-139. doi: 10.1016/j.jim.2013.08. 007

Hirano, T., Taga, T., Yasukawa, K., Nakajima, K., Nakano, N., Takatsuki, F., et al. (1987). Human B-cell differentiation factor defined by an anti-peptide antibody and its possible role in autoantibody production. Proc. Natl. Acad. Sci. U S A 84, 228-231. doi: 10.1073/pnas.84.1.228

Hoffmann, E. K., and Dunham, P. B. (1995). Membrane mechanisms and intracellular signalling in cell volume regulation. Int. Rev. Cytol. 161, 173-262. doi: 10.1016/s0074-7696(08)62498-5

Huang, H., Song, S., Banerjee, S., Jiang, T., Zhang, J., Kahle, K. T., et al. (2019). The WNK-SPAK/OSR1 kinases and the cation-chloride cotransporters as therapeutic targets for neurological diseases. Aging Dis. 10, 626-636. doi: 10. 14336/ad.2018.0928

Hwang, S., Kang, J. Y., Kim, M. J., Shin, D. M., and Hong, J. H. (2019). Carbonic anhydrase 12 mutation modulates membrane stability and volume regulation of aquaporin 5. J. Enzyme Inhib. Med. Chem. 34, 179-188. doi: 10.1080/14756366. 2018.1540475

Ji, M., Kim, H. J., Ahn, C. B., Son, K. H., and Hong, J. H. (2020). Cellular channelopathy mediated by hypergravity: IL-6-mediated Nkccl activation and enhanced Trpm2 expression in rat atrium. Cell Tissue Res.383, 1017-1024. doi: $10.1007 /$ s00441-020-03299-2

Jones, D. S., Jenney, A. P., Swantek, J. L., Burke, J. M., Lauffenburger, D. A., and Sorger, P. K. (2017). Profiling drugs for rheumatoid arthritis that inhibit synovial fibroblast activation. Nat. Chem. Biol. 13, 38-45. doi: 10.1038/ nchembio.2211

Jones, S. A., and Jenkins, B. J. (2018). Recent insights into targeting the IL-6 cytokine family in inflammatory diseases and cancer. Nat. Rev. Immunol. 18, 773-789. doi: 10.1038/s41577-0180066-7

Kaneko, S., Satoh, T., Chiba, J., Ju, C., Inoue, K., and Kagawa, J. (2000). Interleukin- 6 and interleukin-8 levels in serum and synovial fluid of patients with osteoarthritis. Cytokines Cell. Mol. Ther. 6, 71-79. doi: 10.1080/ 13684730050515796

Kokebie, R., Aggarwal, R., Lidder, S., Hakimiyan, A. A., Rueger, D. C., Block, J. A., et al. (2011). The role of synovial fluid markers of catabolism and anabolism in osteoarthritis, rheumatoid arthritis and asymptomatic organ donors. Arthritis Res. Ther. 13:R50.

Kokkonen, H., Soderstrom, I., Rocklov, J., Hallmans, G., Lejon, K., and Rantapaa Dahlqvist, S. (2010). Up-regulation of cytokines and chemokines predates the onset of rheumatoid arthritis. Arthritis Rheum. 62, 383-391.

Lee, D. U., Shin, D. M., and Hong, J. H. (2016). The regulatory role of rolipram on inflammatory mediators and cholinergic/adrenergic stimulationinduced signals in isolated primary mouse submandibular gland cells. Mediators Inflamm. 2016:3745961.

Machado Diaz, A. C., Chico Capote, A., Arrieta Aguero, C. A., Rodriguez Alvarez, Y., Garcia Del, Barco Herrera, D., et al. (2012). Proinflammatory soluble interleukin-15 receptor alpha is increased in rheumatoid arthritis. Arthritis 2012:943156.

Mu, Y. R., Zhou, M. Y., Cai, L., Liu, M. M., and Li, R. (2020). Overexpression of aquaporin 1 in synovium aggravates rat collagen-induced arthritis through regulating beta-catenin signaling: an in vivo and in vitro study. J. Inflamm. Res. 13, 701-712. doi: $10.2147 /$ jir.s271664

Nakahara, H., Song, J., Sugimoto, M., Hagihara, K., Kishimoto, T., Yoshizaki, K., et al. (2003). Anti-interleukin-6 receptor antibody therapy reduces vascular endothelial growth factor production in rheumatoid arthritis. Arthritis Rheum. 48, 1521-1529. doi: 10.1002/art.11143

Nanki, T., Nagasaka, K., Hayashida, K., Saita, Y., and Miyasaka, N. (2001). Chemokines regulate IL- 6 and IL-8 production by fibroblast-like synoviocytes from patients with rheumatoid arthritis. J. Immunol. 167, 5381-5385. doi: 10.4049/jimmunol.167.9.5381

Nishimoto, N., Terao, K., Mima, T., Nakahara, H., Takagi, N., and Kakehi, T. (2008). Mechanisms and pathologic significances in increase in serum interleukin-6 (IL-6) and soluble IL-6 receptor after administration of an antiIL-6 receptor antibody, tocilizumab, in patients with rheumatoid arthritis and Castleman disease. Blood 112, 3959-3964. doi: 10.1182/blood-2008-05-155846

Nowell, M. A., Richards, P. J., Horiuchi, S., Yamamoto, N., Rose-John, S., Topley, N., et al. (2003). Soluble IL-6 receptor governs IL- 6 activity in experimental arthritis: blockade of arthritis severity by soluble glycoprotein 130. J. Immunol. 171, 3202-3209. doi: 10.4049/jimmunol.171.6.3202

O’Brien, J. A., Walters, R. J., Valverde, M. A., and Sepulveda, F. V. (1993). Regulatory volume increase after hypertonicity- or vasoactive-intestinalpeptide-induced cell-volume decrease in small-intestinal crypts is dependent on $\mathrm{Na}(+)-\mathrm{K}(+)-2 \mathrm{Cl}-$ cotransport. Pflugers Arch. 423, 67-73. doi: 10.1007/ bf00374962

Okamoto, H., Yamamura, M., Morita, Y., Harada, S., Makino, H., and Ota, Z. (1997). The synovial expression and serum levels of interleukin-6, interleukin11, leukemia inhibitory factor, and oncostatin $M$ in rheumatoid arthritis. Arthritis Rheum. 40, 1096-1105. doi: 10.1002/art.1780400614

Oliviero, F., Galozzi, P., Ramonda, R., De Oliveira, F. L., Schiavon, F., Scanu, A., et al. (2017). Unusual findings in synovial fluid analysis: a review. Ann. Clin. Lab. Sci. 47, 253-259.

Park, K. J., Kang, S. H., Chae, Y. S., Yu, M. O., Cho, T. H., Suh, J. K., et al. (2010). Influence of interleukin- 6 on the development of peritumoral brain edema in meningiomas. J. Neurosurg. 112, 73-80. doi: 10.3171/2009.4.jns09158

Pedersen, S. F., O'donnell, M. E., Anderson, S. E., and Cala, P. M. (2006). Physiology and pathophysiology of $\mathrm{Na}+\mathrm{H}+$ exchange and $\mathrm{Na}+-\mathrm{K}+-2 \mathrm{Cl}-$ cotransport in the heart, brain, and blood. Am. J. Physiol. Regul. Integr. Comp. Physiol. 291, R1-R25.

Pieraut, S., Lucas, O., Sangari, S., Sar, C., Boudes, M., Bouffi, C., et al. (2011). An autocrine neuronal interleukin- 6 loop mediates chloride accumulation and NKCC1 phosphorylation in axotomized sensory neurons. J. Neurosci. 31, 13516-13526. doi: 10.1523/jneurosci.3382-11.2011

Plebanczyk, M., Radzikowska, A., Burakowski, T., Janicka, I., Musialowicz, U., Kornatka, A., et al. (2019). Different secretory activity of articular and subcutaneous adipose tissues from rheumatoid arthritis and osteoarthritis patients. Inflammation 42, 375-386. doi: 10.1007/s10753-018-0901-9

Ramasamy, R., Payne, J. A., Whang, J., Bergmann, S. R., and Schaefer, S. (2001). Protection of ischemic myocardium in diabetics by inhibition of electroneutral Na+-K+-2Cl- cotransporter. Am. J. Physiol. Heart Circ. Physiol. 281, H515$\mathrm{H} 522$. 
Rao, K. V., Reddy, P. V., Curtis, K. M., and Norenberg, M. D. (2011). Aquaporin-4 expression in cultured astrocytes after fluid percussion injury. J. Neurotrauma 28, 371-381. doi: 10.1089/neu.2010.1705

Sengupta, S., Lorente-Rodriguez, A., Earnest, S., Stippec, S., Guo, X., Trudgian, D. C., et al. (2013). Regulation of OSR1 and the sodium, potassium, two chloride cotransporter by convergent signals. Proc. Natl. Acad. Sci. U.S.A. 110, 18826-18831. doi: 10.1073/pnas.1318676110

Shao, X. T., Feng, L., Gu, L. J., Wu, L. J., Feng, T. T., Yang, Y. M., et al. (2009). Expression of interleukin-18. IL-18BP, and IL-18R in serum, synovial fluid, and synovial tissue in patients with rheumatoid arthritis. Clin. Exp. Med. 9, 215-221. doi: 10.1007/s10238-009-0036-2

Swaak, A. J., Van Rooyen, A., Nieuwenhuis, E., and Aarden, L. A. (1988). Interleukin-6 (IL-6) in synovial fluid and serum of patients with rheumatic diseases. Scand. J. Rheumatol. 17, 469-474.

Takano, T., Ohno, M., Takeuchi, Y., Mandai, R., and Yoshida, S. (2002). Cytotoxic edema and interleukin-6 in hypertensive encephalopathy. Pediatr. Neurol. 26, 71-73. doi: 10.1016/s0887-8994(01)00345-9

Tanaka, T., Narazaki, M., and Kishimoto, T. (2014). IL-6 in inflammation, immunity, and disease. Cold Spring Harb. Perspect. Biol. 6:a016295.

Trujillo, E., Gonzalez, T., Marin, R., Martin-Vasallo, P., Marples, D., and Mobasheri, A. (2004). Human articular chondrocytes, synoviocytes and synovial microvessels express aquaporin water channels; upregulation of AQP1 in rheumatoid arthritis. Histol. Histopathol. 19, 435-444.

Wang, F., Xu, L., Feng, X., Guo, D., Tan, W., and Zhang, M. (2012). Interleukin-29 modulates proinflammatory cytokine production in synovial inflammation of rheumatoid arthritis. Arthritis Res. Ther. 14:R228.

Wang, S., Liang, S., Zhao, X., He, Y., and Qi, Y. (2017). Propofol inhibits cell proliferation and invasion in rheumatoid arthritis fibroblast-like synoviocytes via the nuclear factor-kappaB pathway. Am. J. Transl. Res. 9, 2429-2436.
Wang, T., and He, C. (2020). TNF-alpha and IL-6: the link between immune and bone system. Curr. Drug. Targets 21, 213-227. doi: 10.2174/ 1389450120666190821161259

Xu, W., Mu, X., Wang, H., Song, C., Ma, W., Jolkkonen, J., et al. (2017). Chloride Co-transporter NKCC1 inhibitor bumetanide enhances neurogenesis and behavioral recovery in rats after experimental stroke. Mol. Neurobiol. 54, 2406-2414. doi: 10.1007/s12035-0169819-0

Yue, C., You, X., Zhao, L., Wang, H., Tang, F., Zhang, F., et al. (2010). The effects of adalimumab and methotrexate treatment on peripheral Th17 cells and IL-17/IL-6 secretion in rheumatoid arthritis patients. Rheumatol. Int. 30, 1553-1557.

Conflict of Interest: The authors declare that the research was conducted in the absence of any commercial or financial relationships that could be construed as a potential conflict of interest.

Publisher's Note: All claims expressed in this article are solely those of the authors and do not necessarily represent those of their affiliated organizations, or those of the publisher, the editors and the reviewers. Any product that may be evaluated in this article, or claim that may be made by its manufacturer, is not guaranteed or endorsed by the publisher.

Copyright (C) $2021 \mathrm{Ji}, \mathrm{Ryu}$ and Hong. This is an open-access article distributed under the terms of the Creative Commons Attribution License (CC BY). The use, distribution or reproduction in other forums is permitted, provided the original author(s) and the copyright owner(s) are credited and that the original publication in this journal is cited, in accordance with accepted academic practice. No use, distribution or reproduction is permitted which does not comply with these terms. 